\begin{tabular}{|l|l|}
\hline Postprint Version & 1.0 \\
\hline Journal website & $\underline{\text { http://www.sciencedirect.com }}$ \\
Pubmed link & $\begin{array}{l}\text { http://www.ncbi.nlm.nih.gov/entrez/query.fcgi?cmd=Retrieve\&db=pubmed\&dop } \\
\text { t=Abstract\&list uids=15163485\&query hl=77\&itool=pubmed docsum }\end{array}$ \\
\hline DOI & $\underline{10.1016 / j . v i r u s r e s .2004 .02 .009}$
\end{tabular}

\title{
Harmonising the virological surveillance of influenza in Europe: results of an 18-country survey
}

\author{
T.J. MeERHOFF ${ }^{\text {A, } *}$, W.J. PAget ${ }^{\text {A }}$, J.-F. AgUiLERA ${ }^{\mathrm{B}}$, J. VAN DER VELDEN ${ }^{\mathrm{C}}$
}

a EISS Co-ordination Centre, Netherlands Institute for Health Services Research (NIVEL), Postbus 1568, 3500 BN Utrecht, The Netherlands

b Respiratory Division, Communicable Disease Surveillance Centre (CDSC), PHLS, London, UK c Netherlands School of Public Health, Utrecht, The Netherlands

* Correspondiing author. Tel.: +31-30-27-29-766; fax: +31-30-27-29-729. E-mail address:

t.meerhoff@nivel.nl (T.J. Meerhoff).

\begin{abstract}
The European influenza surveillance scheme (EISS) is based on a surveillance model that combines clinical and virological data in the general population. Eighteen countries in Europe report weekly influenza activity to EISS (http://www.eiss.org). A questionnaire on the virological data collection was sent electronically to the EISS members. Questions on the specimen collection, representativity, laboratory diagnosis of influenza, reporting of variants, testing for other respiratory infections, serological testing, near-patient tests and preparedness for a pandemic were included. The results of the survey showed that for the typing of influenza, the methods polymerase chain reaction (PCR), enzyme-linked immunosorbent assay (ELISA) and haemagglutination inhibition (HAI) methods are used most frequently. For the subtyping of influenza, HAI and PCR are used most often. An east-west divide was seen for the use of PCR in typing and subtyping (more PCR use in the west). Differences in the virological surveillance systems in Europe can make direct comparisons difficult because detection rates may differ by test. For the reporting of other respiratory infections besides influenza, many networks (68\%) collect information on RSV. This is important because RSV causes similar clinical symptoms as influenza and can have a similar impact in terms of burden of disease. With the collection of RSV data, EISS is moving to a broader spectrum of viral respiratory diseases. The findings of this survey will be used to better harmonise laboratory methods in EISS in order to obtain more reliable and comparable information on influenza activity in Europe.
\end{abstract}

\section{INTRODUCTION}

The European influenza surveillance scheme (EISS) was established in 1996. The original members of EISS were: Belgium, France, Germany, The Netherlands, Portugal, Spain and the United Kingdom (EISS, 2002). EISS has gradually grown over the years and had 18 member countries covering 21 influenza surveillance networks during the 2001-2002 influenza season: Belgium, the Czech Republic, Denmark, England, France, Germany, Ireland, Italy, The Netherlands, Northern Ireland, Norway, Poland, Portugal, Scotland, the Slovak Republic, Romania, Slovenia, Spain, Sweden, Switzerland and Wales (Paget et al., 2002). EISS has expanded not only in western Europe, but also (especially in the previous 3 years) in eastern Europe. 
The members of EISS deliver weekly data on influenza activity during the winter season (week 2040). Clinical and virological data is entered into the EISS database and made available on the EISS website (Snacken et al., 1998). This allows the EISS members to query the EISS database, to access maps and find project-related information. Additionally, a weekly electronic bulletin that reports on the influenza activity in Europe is published during the influenza season.

The basis of the scheme is the combined clinical and virological surveillance of influenza in the general population. In EISS, influenza surveillance is based on reports made by sentinel practitioners (SPs), who are general practitioners (GPs) (in all networks), paediatricians (nine networks) and physicians specialised in internal medicine (two networks) (Paget et al., 2002). The SPs usually represent $1-5 \%$ of all practitioners in the country (EISS, 2002). This system an influenza-like illness (ILI) or an acute respiratory infection (ARI) per 100,000 populations. The SPs take nose and/or throat swabs from a sample of the patients with an ILI or ARI and send the swabs to a national reference laboratory where they are tested for influenza.

Besides sentinel influenza test results, the national reference laboratories also report results from nonsentinel sources (e.g. from hospitals, non-sentinel GPs and nursing homes). This non-sentinel data is used to describe better the epidemiology and virology of influenza in the country and to validate the data provided by the SP-based surveillance systems.

Countries participating in the EISS project use different methodologies for the surveillance of influenza (e.g. different case definitions, virological testing methods and specimen collection). This can make it difficult to compare clinical and virological data between countries. In order to improve the data collection procedures, two surveys have been carried out: one on the clinical data collected by EISS (Aguilera et al., 2001) and the other on the virological data (described in this paper).

\section{MATERIALS AND METHODS}

A questionnaire was developed and sent electronically to the EISS collaborating networks (18 countries, 21 networks) in June 2002. Questions on the following topics were included in the questionnaire: specimen collection, representativity, laboratory diagnosis of influenza, reporting of variants, testing for other respiratory infections, serological testing, near-patients tests and preparedness for a pandemic. All of the networks responded. The results on two topics will be presented and discussed in this paper: methods used for typing and subtyping of influenza and testing for other respiratory infections. For the analysis of the methods, the networks were divided into two groups: (1) western Europe (Belgium, Denmark, England, France, Germany, Ireland, Italy, The Netherlands, Norway, Northern Ireland, Portugal, Scotland, Spain, Sweden, Switzerland and Wales), and (2) eastern Europe (Czech Republic, Slovak Republic, Slovenia, Poland and Romania).

\section{RESULTS}

\subsection{Methods used for typing and subtyping of influenza (sentinel specimens)}

The methods used routinely for the typing of influenza (Fig. 1) are polymerase chain reaction (PCR), haemagglutination inhibition (HAI), enzyme-linked immunosorbent assay (ELISA), immunofluorescence (IF) and immunoperoxidase staining (IPT). The bar chart reveals a clear difference between the two groups: PCR is used most often $(60 \% ; n=15)$ by the western countries , while HAI (60\%) and ELISA (60\%) are used most often by the eastern countries. It is important to note that eight networks used more than one technique for the typing of influenza (four west; four east).

\section{[ FIGURE 1 ]}

The methods routinely used for subtyping of influenza (Fig. 2) are HAI, PCR, IF and neuraminidase inhibition (NI). All eastern countries use HAI for the subtyping of influenza, while most (77\%) of the western countries use PCR. Seven networks do more than one test for the subtyping of influenza (six west; one east). 


\section{[ FIGURE 2 ]}

\subsection{Testing for other respiratory infections (sentinel specimens)}

Thirteen out of nineteen networks (68\%) collect information on other viruses (Table 1). All 13 networks collect information on respiratory syncytial virus (RSV), six networks (46\%) collect data on para-influenza virus, five networks (38\%) collect data on adenovirus and three networks (23\%) collect data on rhinovirus. Three networks have information on other viruses (e.g. corona virus, Chlamydia pneumoniae, human metapneumovirus). Eleven out of thirteen (85\%) networks reported that sentinel swabs were tested for both influenza and RSV.

\section{[ TABLE 1 ]}

\section{DISCUSSION}

The survey illustrated the differences in virological data collection among the EISS member countries. The laboratory tests used for typing and subtyping of influenza showed an east-west divide: the eastern European countries use PCR less often than countries in western Europe. The survey also showed that testing for RSV is common, $68 \%$ of networks had sentinel data on RSV.

In general, RT-PCR is (on the condition that an appropriate combination of primers is used and the PCR conditions are optimised) a sensitive and specific method for detecting and typing influenza viruses (Hermann et al., 2001). When you compare RT-PCR with ELISA, PCR has revealed a higher detection rate in some studies (Steininger et al., 2002). It has been suggested that ELISA is reliable for rapid laboratory diagnosis of influenza in infants and young children, but for older patients the use of RT-PCR is necessary to avoid false negative reactions (Steininger et al., 2002).

Different techniques show different sensitivities. The use of ELISA by the eastern European countries may lead to an underestimation of influenza activity due to the occurrence of false negative samples and may affect the early warning function of their networks and EISS. The reason for using ELISA instead of PCR is probably due to fewer resources available for influenza surveillance in eastern European countries. PCR requires specialised equipment and the cost per sample is relatively high.

Not only were differences found in the techniques used (PCR versus ELISA), but there were also differences in the way the methods are used. This has been illustrated by the quality control assessment (QCA) that was initiated in 2000-2001 to evaluate the quality of the influenza and RSV testing in 16 national reference laboratories in EISS (Vallette and Aymard, 2002). In this study, the sensitivity of the RT-PCR varied widely (40-100\% for influenza, $71-86 \%$ for RSV) depending on the laboratory (within the same laboratory the sensitivity was identical to that of cell culture).

The clinical symptoms and the impact of RSV can be similar to that of influenza. The burden of disease caused by RSV has led to plans for the development of RSV vaccines. Within EISS, networks can enter virological results on RSV testing into the EISS database. The survey illustrated that many (68\%) networks collect sentinel RSV data, of which 85\% test the sentinel swabs for both influenza and RSV. This result shows that moving to a broader spectrum of respiratory infections besides influenza seems feasible.

In order to improve the comparability and validity of results, EISS needs to harmonise viral procedures and data collection methods (e.g. sensitivity of the methods used, use of standardised reagents, quality of the samples collected, reporting of RSV). Harmonisation of methods in general and within the method itself (e.g. PCR) is also very important. The results of this survey will be used to better harmonise laboratory methods in EISS in order to obtain more reliable and comparable information on influenza activity in Europe. 
Meerhoff, T.J., Paget, W.J., Aguilera, J.F., Velden, J. van der

Harmonising the virological surveillance of influenza in Europe: results of an 18-country survey.

Virus Research: 103, 2004, p. 31-33

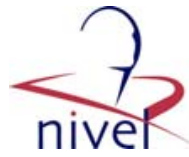

\section{TABLES AND FIGURES}

Table 1

Collection of sentinel RSV data by the EISS networks

\begin{tabular}{lll}
\hline RSV data collected & Percentage & Networks \\
\hline Yes & $68(13 / 19)$ & $\begin{array}{l}\text { Czech Republic, England, France, Germany, The Netherlands, Northern Ireland, Portugal, Romania (Bucharest), } \\
\text { Scotland, Slovenia, Spain, Switzerland, Wales } \\
\text { No }\end{array}$ \\
\hline
\end{tabular}

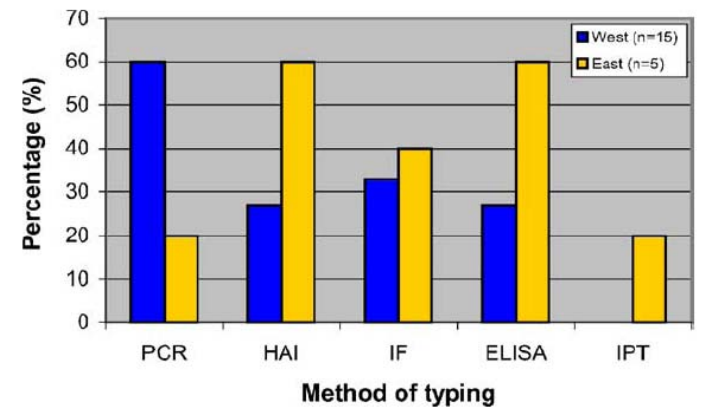

Fig. 1. Methods used for typing of influenza by the EISS networks (sen- Fig. 2. Methods used for subtyping of influenza by the EISS networks tinel specimens, total percentage $>100$ when more than one method is (sentinel specimens, total percentage $>100$ when more than one method used for typing of influenza). West $(n=15)$ : Belgium, Denmark, Eng- is used for subtyping of influenza). West $(n=13)$ : Belgium, Denmark, land, France, Germany, Ireland, Italy, The Netherlands, Northern Ireland, England, France, Germany, Ireland, Italy, The Netherlands, Northern IreNorway, Portugal, Scotland, Spain, Switzerland and Wales. East $(n=5)$ : land, Norway, Portugal, Spain and Switzerland. East $(n=5)$ : Czech Czech Republic, Slovak Republic, Slovenia, Poland and Romania.

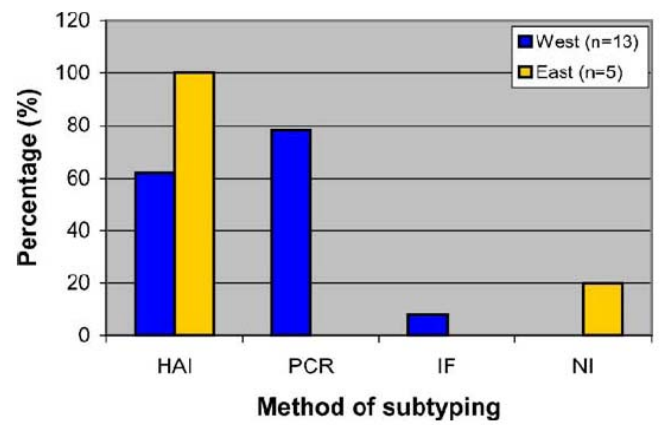

land, Norway, Portugal, Spain and Switzerland. East $(n$
Republic, Slovak Republic, Slovenia, Poland and Romania.

\section{REFERENCES}

1. Aguilera, J.-F., Paget, W.J., Manuguerra, J.-C., on behalf of European influenza surveillance scheme (EISS) and EuroGROG (2001). Survey of influenza surveillance systems in Europe. Report.

2. European influenza surveillance scheme, 2002. Annual Report: 2000-2001 influenza season. Utrecht, The Netherlands: NIVEL (can be downloaded from the EISS website).

3. Hermann, B., Larsson, C., Wirgart, B.Z., 2001. Simultaneous detection and typing of influenza viruses $A$ and $B$ by a nested reverse transcription-PCR: comparison to virus isolation and antigen detection by immunofluorescence and optical immunoassay (FLU OIA). J. Clin. Microbiol. 39 (1), 134-138.

4. Paget, W.J., Meerhoff, T.J. and Goddard, N.L., on behalf of EISS (2002). Mild to moderate influenza activity in Europe and the detection of novel $A(H 1 N 2)$ and $B$ viruses during the winter of 2001-2002. Eurosurveillance 7, 147-157.

5. Snacken, R., Manuguerra, J.C., Taylor, P., 1998. European influenza surveillance scheme on the internet. Methods Inf. Med. 37, 266-270.

6. Steininger, C., Kundi, M., Aberle, S.W., Aberle, J.H., Popow-Kraubb, T., 2002. Effectiveness of reverse transcription-PCR, virus isolation, and enzyme-linked immunosorbent assay for diagnosis of influenza A virus infection in different age groups. J. Clin. Microbiol. 40 (6), 20512056.

7. Vallette, M., Aymard, M., 2002. Quality control assessment of influenza and RSV testing in Europe: 2000-2001 season. Eurosurveillance 7, 161-165. 